\title{
Shape symmetrization and IR-spectra enhancement of aluminum clusters via doping with a carbon core
}

\author{
Fedor Y. Naumkin \\ Faculty of Science, Ontario Tech University / UOIT, Oshawa, ON L1G 0C5, Canada
}

\section{Supplementary information}

DFT-PBE0/cc-pVTZ optimized energies (in a.u.) and atomic xyz coordinates $(\AA)$ of the studied species

\begin{tabular}{|c|c|c|c|c|}
\hline \multicolumn{5}{|c|}{$E=-3877.47265923$} \\
\hline $1 \mathrm{Al}$ & 13.0000 & 1.39543443 & 0.31681616 & 1.03414406 \\
\hline $2 \mathrm{Al}$ & 13.0000 & 1.96324989 & 0.45427140 & -1.89122725 \\
\hline $3 \mathrm{Al}$ & 13.0000 & -1.38860012 & -0.32248044 & 1.03879033 \\
\hline $4 \mathrm{Al}$ & 13.0000 & -1.97475906 & -0.45041727 & -1.89031308 \\
\hline $5 \mathrm{Al}$ & 13.0000 & 1.74939279 & 2.80836036 & 0.00020263 \\
\hline $6 \mathrm{Al}$ & 13.0000 & -2.80986103 & 1.75116728 & 0.01265239 \\
\hline $7 \mathrm{Al}$ & 13.0000 & 2.81115442 & -1.74939329 & -0.01631419 \\
\hline $8 \mathrm{Al}$ & 13.0000 & -1.75055737 & -2.81167939 & 0.00600411 \\
\hline $9 \mathrm{Al}$ & 13.0000 & -0.44670035 & 1.96666560 & 1.88930549 \\
\hline $10 \mathrm{Al}$ & 13.0000 & 0.45622802 & -1.97112730 & 1.89115046 \\
\hline $11 \mathrm{Al}$ & 13.0000 & -0.32557642 & 1.39337296 & -1.03675168 \\
\hline $12 \mathrm{Al}$ & 13.0000 & 0.31473468 & -1.39176091 & -1.03797689 \\
\hline $13 \mathrm{Al}$ & 13.0000 & 3.75725114 & 0.87434358 & -0.03395310 \\
\hline $14 \mathrm{Al}$ & 13.0000 & -3.75362305 & -0.87271675 & -0.01991608 \\
\hline $15 \mathrm{Al}$ & 13.0000 & -0.87263862 & 3.75775740 & 0.03113666 \\
\hline & 13. & 0.8748 & -3.7531793 & 0.02306 \\
\hline
\end{tabular}

Al18

$E=-4362.22060195$

\begin{tabular}{|c|c|c|c|c|}
\hline & 13.0000 & 1.48018674 & -0.45042677 & \\
\hline & 13.0000 & 2.02224783 & -0.61538259 & \\
\hline & 13.00 & -1.47993835 & 0.45047452 & \\
\hline & 13.0000 & -2.02229534 & 0.61547725 & \\
\hline
\end{tabular}




$\begin{array}{lcrrl}5 \mathrm{Al} & 13.0000 & 1.57508550 & -2.95401192 & 0.00002402 \\ 6 \mathrm{Al} & 13.0000 & -2.95378674 & -1.57543620 & -0.00007570 \\ 7 \mathrm{Al} & 13.0000 & 2.95418184 & 1.57512739 & 0.00013212 \\ 8 \mathrm{Al} & 13.0000 & -1.57564355 & 2.95394262 & -0.00000146 \\ 9 \mathrm{Al} & 13.0000 & -0.61528273 & -2.02215179 & -1.81882537 \\ 10 \mathrm{Al} & 13.0000 & 0.61554976 & 2.02222127 & -1.81905191 \\ 11 \mathrm{Al} & 13.0000 & -0.45036540 & -1.47985419 & 1.11021095 \\ 12 \mathrm{Al} & 13.0000 & 0.45043305 & 1.48009625 & 1.10986865 \\ 13 \mathrm{Al} & 13.0000 & 3.73460360 & -1.13704805 & -0.00261071 \\ 14 \mathrm{Al} & 13.0000 & -3.73468393 & 1.13643126 & -0.00288421 \\ 15 \mathrm{Al} & 13.0000 & -1.13685467 & -3.73443601 & 0.00275724 \\ 16 \mathrm{Al} & 13.0000 & 1.13665118 & 3.73476703 & 0.00233209 \\ 17 \mathrm{Al} & 13.0000 & -0.00002386 & -0.00003466 & -3.19298877 \\ 18 \mathrm{Al} & 13.0000 & -0.00006492 & 0.00024459 & 3.19321235\end{array}$

C8@Al16 $(\mathrm{S}=1)$

$E=-4181.63788297$

$\begin{array}{lcccc}1 \mathrm{C} & 6.0000 & 0.78849457 & -0.66000403 & 0.90966325 \\ 2 \mathrm{C} & 6.0000 & 0.78767269 & -0.90005740 & -0.66890258 \\ 3 \mathrm{C} & 6.0000 & 0.79014170 & 0.89943239 & 0.67264402 \\ 4 \mathrm{C} & 6.0000 & 0.78938220 & 0.65891091 & -0.90576895 \\ 5 \mathrm{C} & 6.0000 & -0.78868364 & -0.65821551 & 0.91018408 \\ 6 \mathrm{C} & 6.0000 & -0.78900681 & -0.89854429 & -0.66808509 \\ 7 \mathrm{C} & 6.0000 & -0.78724410 & 0.90075780 & 0.67219789 \\ 8 \mathrm{C} & 6.0000 & -0.78739828 & 0.66019637 & -0.90602300 \\ 9 \mathrm{Al} & 13.0000 & -0.00085393 & -1.82523389 & 2.57669232 \\ 10 \mathrm{Al} & 13.0000 & -0.00228491 & -2.50516967 & -1.91793600 \\ 11 \mathrm{Al} & 13.0000 & -0.00238374 & 2.50895725 & 1.91729631 \\ 12 \mathrm{Al} & 13.0000 & 0.00713824 & 1.82108519 & -2.57655974 \\ 13 \mathrm{Al} & 13.0000 & 2.19403744 & -2.17644366 & 0.32990712 \\ 14 \mathrm{Al} & 13.0000 & 2.19948475 & 2.17131531 & -0.32766915 \\ 15 \mathrm{Al} & 13.0000 & -2.19904640 & -2.16938104 & 0.33350535 \\ 16 \mathrm{Al} & 13.0000 & -2.19438601 & 2.17436372 & -0.33465780 \\ 17 \mathrm{Al} & 13.0000 & 2.18623356 & 0.34119148 & 2.26179648 \\ 18 \mathrm{Al} & 13.0000 & -2.18258425 & 0.34959286 & 2.26336403 \\ 19 \mathrm{Al} & 13.0000 & 2.17896706 & -0.34528226 & -2.26431182 \\ 20 \mathrm{Al} & 13.0000 & -2.18215638 & -0.34389231 & -2.26005179 \\ 21 \mathrm{Al} & 13.0000 & -0.00525134 & -3.75600433 & 0.56978441 \\ 22 \mathrm{Al} & 13.0000 & 0.00400261 & 3.75505702 & -0.57290428 \\ 23 \mathrm{Al} & 13.0000 & 3.79074597 & -0.00569618 & -0.00464659 \\ 24 \mathrm{Al} & 13.0000 & -3.79223202 & 0.00442086 & 0.00137139\end{array}$




\begin{tabular}{ccccc}
$\mathbf{C} @ \mathbf{8} \mathbf{A l} \mathbf{1 8}$ & \multicolumn{5}{l}{} \\
$\begin{array}{c}\mathrm{E}=-4666.40205452 \\
\end{array}$ & & & & \\
$1 \mathrm{C}$ & 6.0000 & 0.80001178 & 0.82700556 & 0.77516431 \\
$2 \mathrm{C}$ & 6.0000 & 0.82676726 & -0.80047629 & 0.77491418 \\
$3 \mathrm{C}$ & 6.0000 & 0.80067876 & 0.82722019 & -0.77429425 \\
$4 \mathrm{C}$ & 6.0000 & 0.82746346 & -0.80021022 & -0.77461669 \\
$5 \mathrm{C}$ & 6.0000 & -0.82746346 & 0.80021022 & 0.77461669 \\
$6 \mathrm{C}$ & 6.0000 & -0.80067876 & -0.82722019 & 0.77429425 \\
$7 \mathrm{C}$ & 6.0000 & -0.82676726 & 0.80047629 & -0.77491418 \\
$8 \mathrm{C}$ & 6.0000 & -0.80001178 & -0.82700556 & -0.77516431 \\
$9 \mathrm{Al}$ & 13.0000 & -0.03893287 & 2.32002834 & 2.08939835 \\
$10 \mathrm{Al}$ & 13.0000 & 0.03752530 & -2.32019109 & 2.08915149 \\
$11 \mathrm{Al}$ & 13.0000 & -0.03752530 & 2.32019109 & -2.08915149 \\
$12 \mathrm{Al}$ & 13.0000 & 0.03893287 & -2.32002834 & -2.08939835 \\
$13 \mathrm{Al}$ & 13.0000 & 2.31908463 & 0.03764517 & 2.09034544 \\
$14 \mathrm{Al}$ & 13.0000 & 2.32114875 & 0.03880952 & -2.08824701 \\
$15 \mathrm{Al}$ & 13.0000 & -2.32114875 & -0.03880952 & 2.08824701 \\
$16 \mathrm{Al}$ & 13.0000 & -2.31908463 & -0.03764517 & -2.09034544 \\
$17 \mathrm{Al}$ & 13.0000 & 2.19838336 & 2.27271415 & 0.00126630 \\
$18 \mathrm{Al}$ & 13.0000 & -2.27270325 & 2.19838868 & -0.00027433 \\
$19 \mathrm{Al}$ & 13.0000 & 2.27270325 & -2.19838868 & 0.00027433 \\
$20 \mathrm{Al}$ & 13.0000 & -2.19838336 & -2.27271415 & -0.00126630 \\
$21 \mathrm{Al}$ & 13.0000 & -0.00173876 & -0.00004577 & 3.44651005 \\
$22 \mathrm{Al}$ & 13.0000 & 0.00173876 & 0.00004577 & -3.44651005 \\
$23 \mathrm{Al}$ & 13.0000 & 4.14030330 & 0.06907621 & 0.00194035 \\
$24 \mathrm{Al}$ & 13.0000 & -4.14030330 & -0.06907621 & -0.00194035 \\
$25 \mathrm{Al}$ & 13.0000 & -0.06908371 & 4.14045868 & 0.00019684 \\
$26 \mathrm{Al}$ & 13.0000 & 0.06908371 & -4.14045868 & -0.00019684
\end{tabular}

\section{$\mathrm{C}_{8 A 1}{ }^{(\mathrm{c})}$}

$E=-4181.71378574$

$\begin{array}{lcccc}1 \mathrm{C} & 6.0000 & -0.00411253 & -3.06420823 & 1.14796325 \\ 2 \mathrm{C} & 6.0000 & 1.10436846 & -3.06693993 & 0.00576059 \\ 3 \mathrm{C} & 6.0000 & -1.10334265 & -3.07774589 & -0.00326717 \\ 4 \mathrm{C} & 6.0000 & 0.00502134 & -3.06547332 & -1.14540747 \\ 5 \mathrm{C} & 6.0000 & 0.00323594 & -4.68799301 & 1.18020403 \\ 6 \mathrm{C} & 6.0000 & 0.86393363 & -4.55985542 & 0.00487825 \\ 7 \mathrm{C} & 6.0000 & -0.84900764 & -4.56837539 & -0.00239630 \\ 8 \mathrm{C} & 6.0000 & 0.01308698 & -4.68961416 & -1.17826220 \\ 9 \mathrm{Al} & 13.0000 & -1.96054106 & 2.58594706 & -0.00257232 \\ 10 \mathrm{Al} & 13.0000 & -2.06332801 & -1.33951072 & -0.00405039\end{array}$




$\begin{array}{lrrrr}11 \mathrm{Al} & 13.0000 & 1.95918949 & 2.58846072 & 0.00060788 \\ 12 \mathrm{Al} & 13.0000 & 2.05965476 & -1.32497955 & 0.00700530 \\ 13 \mathrm{Al} & 13.0000 & -1.56075135 & 0.63036564 & 2.11602026 \\ 14 \mathrm{Al} & 13.0000 & 1.55535198 & 0.62354898 & 2.09299413 \\ 15 \mathrm{Al} & 13.0000 & -1.55605334 & 0.62262191 & -2.11921338 \\ 16 \mathrm{Al} & 13.0000 & 1.56305519 & 0.62362967 & -2.08760942 \\ 17 \mathrm{Al} & 13.0000 & -0.00199395 & 2.72957312 & 1.84615841 \\ 18 \mathrm{Al} & 13.0000 & -0.00087040 & 2.72312401 & -1.84659250 \\ 19 \mathrm{Al} & 13.0000 & -0.00882024 & -1.40427099 & 2.18105698 \\ 20 \mathrm{Al} & 13.0000 & 0.00644065 & -1.40641577 & -2.17909927 \\ 21 \mathrm{Al} & 13.0000 & -3.70486115 & 0.68372247 & -0.00957859 \\ 22 \mathrm{Al} & 13.0000 & 3.72107801 & 0.69446893 & 0.00227628 \\ 23 \mathrm{Al} & 13.0000 & -0.00291932 & 4.72714952 & -0.00421926 \\ 24 \mathrm{Al} & 13.0000 & -0.01967461 & 0.45206003 & 0.00051334\end{array}$

\section{$\mathrm{C}_{8} \mathrm{Al}_{18}{ }^{(\mathrm{c})}$}

$E=-4666.42593762$

$\begin{array}{lcccc}1 \mathrm{C} & 6.0000 & 1.15503079 & 3.05994925 & -0.00459140 \\ 2 \mathrm{C} & 6.0000 & 0.00607875 & 3.07739293 & 1.09135386 \\ 3 \mathrm{C} & 6.0000 & -0.00271618 & 3.07848659 & -1.09103151 \\ 4 \mathrm{C} & 6.0000 & -1.15204800 & 3.06411938 & 0.00475770 \\ 5 \mathrm{C} & 6.0000 & 1.19054519 & 4.69603909 & -0.00366585 \\ 6 \mathrm{C} & 6.0000 & 0.00834783 & 4.56826553 & 0.84840507 \\ 7 \mathrm{C} & 6.0000 & 0.00166146 & 4.56924498 & -0.84674661 \\ 8 \mathrm{C} & 6.0000 & -1.17993741 & 4.70031058 & 0.00552339 \\ 9 \mathrm{Al} & 13.0000 & -0.01074581 & -2.52151717 & -1.96422198 \\ 10 \mathrm{Al} & 13.0000 & -0.01151473 & 1.42228824 & -2.16282545 \\ 11 \mathrm{Al} & 13.0000 & 0.00839110 & -2.51797131 & 1.96204492 \\ 12 \mathrm{Al} & 13.0000 & 0.00782663 & 1.42287178 & 2.16610025 \\ 13 \mathrm{Al} & 13.0000 & 1.87399734 & -0.47590434 & -1.88453888 \\ 14 \mathrm{Al} & 13.0000 & 1.89135525 & -0.47575426 & 1.86641789 \\ 15 \mathrm{Al} & 13.0000 & -1.89540804 & -0.47252801 & -1.86804639 \\ 16 \mathrm{Al} & 13.0000 & -1.87750345 & -0.47337029 & 1.88373454 \\ 17 \mathrm{Al} & 13.0000 & 1.94776589 & -2.54590453 & -0.00814389 \\ 18 \mathrm{Al} & 13.0000 & -1.95166815 & -2.54663280 & 0.01039341 \\ 19 \mathrm{Al} & 13.0000 & 2.23360363 & 1.39420278 & -0.01103845 \\ 20 \mathrm{Al} & 13.0000 & -2.23179274 & 1.39801281 & 0.00833001 \\ 21 \mathrm{Al} & 13.0000 & -0.01914916 & -0.66934412 & -3.79694819 \\ 22 \mathrm{Al} & 13.0000 & 0.01580741 & -0.66709063 & 3.80028705 \\ 23 \mathrm{Al} & 13.0000 & 3.80891336 & -0.73756024 & -0.01952355 \\ 24 \mathrm{Al} & 13.0000 & -3.80872898 & -0.73656859 & 0.01684135 \\ 25 \mathrm{Al} & 13.0000 & -0.00192967 & -4.59407903 & -0.00052953 \\ 26 \mathrm{Al} & 13.0000 & -0.00147843 & -0.37341919 & -0.00055720\end{array}$

